IDDF2019-ABS-0285 MANAGEMENT OF CAUSTIC CONSUMPTION INJURIES - A MULTIDISCIPLINARY EFFORT FOR A SUCCESSFUL OUTCOME

Tripuraneni Venkata Aditya Chowdary*, Nara Bharat Kumar. Gleneagles Global Hospital, India

10.1136/gutjnl-2019-IDDFabstracts.223

Background Ingestion of corrosive substances results in severe damage of the upper aero digestive system and is still a major cause of concern in India. Injuries depend on the type of substance, quality, quantity and intention. Corrosive agents produce extensive damage to the gastrointestinal tract, which may result in death in the acute phase or may result in long-term sequelae. We describe the experience of our department in treating such injuries.

Methods All corrosive injury patients, acute and late, presented to the Department of Surgical Gastroenterology from Jan 2009 - May 2018, are included in this retrospective study.

Results Between Jan 2009 - May 2018, 85 patients were admitted with a history of corrosive agent consumption. $\underline{48}$ patients had an only mild mucosal injury and were treated conservatively and required no further intervention.

4 required emergency total gastrectomy with a cervical esophagostomy of whom only one survived.

26 patients underwent repeated esophageal dilatations for strictures. 1 of them had a spontaneous duodenal perforation and succumbed. 19 patients responded very well to the dilatation protocol. $\underline{6}$ patients underwent surgery for complication/ failure of endoscopic dilatation.

7 patients presented late with well-established strictures with poor nutrition and underwent a feeding jejunostomy with a reconstructive procedure at a later date.

A total of 13 patients underwent a reconstructive procedure, 7 underwent a colonic pull through, 2 gastric pull up and 4 underwent a Bilroth II distal gastrectomy.

Conclusions Corrosive injury of the upper gastrointestinal tract is a complex condition, requiring an intensive approach and multidisciplinary management. Maintenance of nutrition is essential for a good outcome. The native esophagus should be salvaged whenever possible. Meticulous intra-operative technique and ensuring good vascular supply of the conduit are the cornerstones of the successful outcome.

\section{IDDF2019-ABS-0286 DIFFERENCES IN PRESENTATION OF PRIMARY VS STUMP GASTRIC CARCINOMAS: SHORT TERM ANALYSIS}

Palanati Varun*. Narayana Medical College and General Hospital, India

10.1136/gutjnl-2019-IDDFabstracts.224

Background With the advent of effective medical treatmentPPI and H. pylori eradication, surgeries for Peptic Ulcer Disease (PUD) have drastically declined.

Patients who underwent such surgeries a few decades earlier are still encountered in clinical practice, either benign or malignant.

Presentation varies with the type of previous surgery done. Gastric Stump Carcinoma (GSC)- Increased risk of malignancy after gastrectomy, Carcinoma of the gastric remnant or the stump after partial gastrectomy with risk increasing 15 years after gastrectomy.
Previous studies have demonstrated that rates of gastric stump carcinoma are consistently higher after treatment with a Billroth II procedure $>>$ Billroth I procedure

Aim To analyze whether any difference exists between presentation and management of primary gastric carcinoma and stump carcinoma

Methods Retrospective study

patients are divided into 2 groups

I- Patients with GSC following truncal vagotomy and gastrojejunostomy for PUD

II-Patients without previous gastric surgery with primary gastric carcinoma

Clinico-pathological parameters of these groups were compared and analyzed

Inclusion criteria - All patients with Resectable gastric and stump carcinomas in previous $\mathrm{TV}+\mathrm{GJ}$ and good performance status

Results Stomal malignancies presented with GI bleed in all cases(100\%), followed by weight loss (75\%) and vomiting (25\%).Non-stomal malignancies presented with anorexia (83.3\%), GI bleed (75\%) and weight loss (75\%).

Total gastrectomy done in 1 stomal \& 7 non-stomal malignancies. Subtotal gastrectomy done in 3 stomal and $\underline{5 \text { non sto- }}$ mal malignancies.

3 in T3 and 1 in T1 stage in stomal group, 2 in NO and N1 stage each. In non stomal group T4 $(n=7), N 1 \quad(n=7)$ and $\mathrm{N} 2(\mathrm{n}=5)$ presentation.

Conclusions High suspicion for malignancy and low threshold for endoscopy in patients with previous gastrojejunostomy provide an opportunity for early diagnosis and curative resection in stump carcinomas

Altered lymph flow and anatomic relationship with adjacent organs due to initial surgery. Surgical resection is considered an effective therapeutic strategy for GSC. Invasive extent and surgical curability of GSC-Prognosis of patients

GSC have decreased rate of curative resection and longterm survival rate if identified at an advanced stage due to unapparent symptom and High incidence of lymph node metastasis

\section{IDDF2019-ABS-0291 MODIFIED PULLEY TRACTION SYSTEM IN ENDOSCOPIC SUBMUCOSAL DISSECTION (ESD) OF COLONIC LESIONS}

${ }^{1}$ Mann Yie Thian*, ${ }^{2}$ Yoji Takeuchi, ${ }^{2}$ Noriya Uedo, ${ }^{1}$ Stephen Tsao. ${ }^{1}$ Department of Gastroenterology and Hepatology, Tan Tock Seng Hospital, Singapore; ${ }^{2}$ Department of Gastrointestinal Oncology, Osaka International Cancer Institute, Japan

\subsection{6/gutjnl-2019-IDDFabstracts.225}

Background ESD is a proven and safe technique for endoscopic treatment of early gastrointestinal tract cancers. Technical challenges remain especially when resecting large lesions in difficult locations. Traction system providing unilateral traction has been previously described as a useful tool. We present a case series of using the modified pulley traction system in assisting ESD in colonic lesions.

Methods Dental floss was used as a traction line. It was inserted through the biopsy channel and a loop was tied outside the endoscope before starting the procedure. Circumferential incision with Flush Knife BT $1.5 \mathrm{~mm}$ Fujifilm(R) was carried out at the targeted lesion after submucosal lifting. The dental floss loop outside the endoscope was cut and tied to an endoclip which was then placed over the lesion's distal 